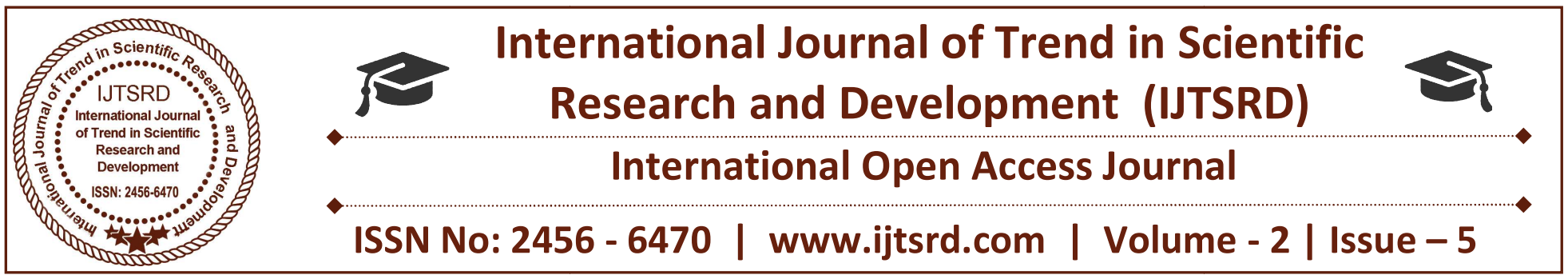

\title{
Protein Energy Malnutrition in CKD Patients
}

\author{
Suraj Kumar Singh, Atul Verma, Hulsi Sahu \\ Dialysis Technologist, \\ Pt. Jawahar Lal Nehru Memorial Medical College, \\ Raipur, Chhattisgarh, India
}

\begin{abstract}
Elevated protein catabolism and protein malnutrition are common in patients with chronic kidney disease (CKD) and end-stage renal disease (ESRD). The underlying etiology includes, but is not limited to, metabolic acidosis intestinal dysbiosis; systemic inflammation with activation of complements, endothelin-1 and renin-angiotensin-aldosterone (RAAS) axis; anabolic hormone resistance; energy expenditure elevation; and uremic toxin accumulation. All of these derangements can further worsen kidney function, leading to poor patient outcomes. Many of these CKD-related derangements can be prevented and substantially reversed, representing an area of great potential to improve CKD and ESRD care. This review integrates known information and recent advances in the area of protein nutrition and malnutrition in CKD and ESRD. Management recommendations are summarized. Thorough understanding the pathogenesis and etiology of protein malnutrition in CKD and ESRD patients will undoubtedly facilitate the design and development of more effective strategies to optimize protein nutrition and improve outcomes.
\end{abstract}

Keywords: protein nutrition; protein catabolism; chronic kidney disease; dialysis; acidosis; inflammation; hormonal derangements; uremic toxins

\section{INTRODUCTION}

Chronic kidney disease (CKD) is currently a public health problem. CKD is a slow, progressive, and irreversible loss of kidney function. Because this loss is slow and progressive, it results in an adaptive process in which the patient remains asymptomatic for some time. However, when the kidneys can no longer adequately remove the metabolic degradation products, dialysis treatment should be initiated. Hem dialysis (HD) is the most common renal treatment today. Despite the benefits of HD in prolonging the survival of patients with CKD, the conditions imposed by the disease and dialysis therapy result in a series of organic changes, with acute and chronic complications and nutritional changes. Additionally, dialysis treatment is associated with high rates of hospitalization and increased mortality. Individuals undergoing dialysis have a significant prevalence of malnutrition, which is classified as mild, moderate, and severe. The cause of malnutrition is multifactorial and includes: inadequate food intake, hormonal and gastrointestinal disorders, dietary restrictions, drugs that alter nutrient absorption, insufficient dialysis, and constant presence of associated diseases. Furthermore, uremia, acidosis, and HD procedure per se are hyper catabolic and associated with the presence of an inflammatory state. Malnutrition is considered a marker of poor prognosis in CKD.

\section{Aims and Objectives:}

$>$ To determine the prevalence of malnutrition among CKD patient on hemodialysis.

$>$ To determine the impact of demographic socioeconomic factors on malnutrition indicators.

$>$ To determine the prevalence of chronic kidney disease complications.

$>$ To evaluate the diet \& fluid compliance among hemodialysis patients.

And to clarify the correlation between dietary intake and malnutrition among hemodialysis patients. 


\section{MATERIALS AND METHODS}

Location of the study The hospital based study was carried out at the hemodialysis unit of DR. B. R. A. Memorial Hospital Raipur, with the purpose to observe and undertake the protocol followed in the hospital to assess the nutritional status of the patients visiting for hemodialysis and different wards for the treatment of chief complaints and associated co morbidities.

\section{Sample Size:-}

A convenient sample of 50 adult hemodialysis patients from both sexes was selected for the study.

\section{Study Setting}

The study was conducted at the hemodialysis unit at Al-Shifa hospital, in Gaza strip, which is considered the dialysiscenter in Dr. B. R. A. Memorial hospital raipur with 4 machines and more than 100 patients.

\section{Study population}

All hemodialysis patients from both gender diagnosed as an ESRD on hemodialysis for more than six months.

\section{Eligibility Criteria}

\section{$>$ Inclusion criteria:-}

$>$ Patients with ESRD from both gender aged 19-59 years.

$>$ On regular hemodialysis for at least six months or more.

\section{$>$ Exclusion criteria:-}

$>$ Patients with other types of acute illness, such as pneumonia, acute myocardial infarction or septicemia.

$>$ Patients with depression.

$>$ Patients $<19$ and $>59$ years old.

$>$ Patients on hemodialysis for $<6$ months.

\section{Collection of data}

Demographic and socioeconomic data Data regarding socioeconomic status like occupation, marital status, education, family type, family size and monthly family income was collected by interviewing the subjects.

\section{Questionnaire interview}

Face to face structured interviews was used to collect data from individuals. Most questions are one of two types: the multiple choice question which offers several fixed alternatives and yes or no question which offers a dichotomous choice. The interviewer explained to all individuals the importance, aim and purpose of the research study. Also all questions were ideally asked in the same way during the data collection to achieve a high degree of validity and reliability.

\section{Diet and fluid compliance data}

Also, the following data concerning diet and fluid compliance was collected from patients files and by using questionnaire interview: using of diet regimen, fluid intake, average weight change between hemodialysis sessions $(\mathrm{kg})$ in the last two months, etc.

\section{Medical history and chief complaints:-}

Questionnaire regarding health status was filled from the subjects and their attendants. After being examined by the consultant, their chief complaints and medical history were collected from the recorded data in patient's file, patient himself/herself or their attendant and details were recorded.

Anthropometric data:- Anthropometric parameters included, body weight, height, arm circumference, body weight was measured using a personal weighing machine (beam balanced scale) before taking the measurement machine was placed on a leveled surface and set at zero. Subjects were asked to stand straight, relaxed and with minimum clothing. Height of the subjects was taken in a standing position, without footwear.

Dietary assessment:- Dietary assessment was done using, 24- hour dietary recall and 3-day diet diary .Standard sized measured utensils (glass, bowl and different circled sized paper board like chapatti) were used. Different type of fat and protein sources being included in diet was assessed.

Laboratory parameters:- The biochemical parameters like serum creatinine (alkaline picrate method), cholesterol (enzymatic end point method), albumin (Bromocresol- Green end point method); All the above tests were done by the fully automatic analyzer(RFCL, Flexor - XL) GFR based on age, weight and creatinine was calculated separately for men and women by using the following formula mentioned below (Cockroft- Gault equation). The cases with GFR greater or equal to 120 were considered as normal. GFR for men $=\left((140 \text {-age })^{*}\right.$ weight $) /(72 *$ creatinine $(\mathrm{mg} / \mathrm{dl}))$; GFR for women $=$ $(((140$-age $) *$ weight $) /(72 *$ creatinine $(\mathrm{mg} / \mathrm{dl}))) * 0.85$ 
Protein Energy Wasting (PEW) assessment:- PEW was diagnosed according to the criteria proposed by the ISRNM 13. These criteria are comprised of four categories and PEW is present if the patient satisfies at least one criteria, in three of the four categories. The criteria from each category applied in this study are as follows:

1. Biochemical: Serum albumin $<3.8 \mathrm{~g} / \mathrm{dl}$ or cholesterol $<100 \mathrm{mg} / \mathrm{dl}$;

2. Body mass: Total body fat percentage $<10 \%$ or unintentional weight loss over time $>10 \%>6$ months;

3. Muscle mass: Standard MAMC $<90 \%$ in relation to national Health and nutrition examination.

4. Dietary intake: unintentional low energy and protein intake (energy: $<25 \mathrm{kcal} / \mathrm{kg} /$ day and protein : $<0.6 \mathrm{gm} / \mathrm{kg} /$ day).

\section{Result:}

1. Distribution of the study sample by biochemical indicators of malnutrition

The National Kidney Foundation Guidelines on CKD recommends that:

1. Serum Albumin $(<4 \mathrm{~g} / \mathrm{dL})$.
2. Serum Creatinine $(<10 \mathrm{mg} / \mathrm{dL})$.

3. Serum Cholesterol $(<165 \mathrm{mg} / \mathrm{dL})$.

As clinically valid indicators in assessing the prevalence of malnutrition in hemodialysis patients (NKF/KDOQI, 2002).

Patients with biochemical indicators of malnutrition. The obtained results shows that the majority of the patients $(58.0 \%)$ had serum albumin $<4.0 \mathrm{~g} / \mathrm{dL}, 64.0 \%$ with serum creatinine $<10 \mathrm{mg} / \mathrm{dL}$ and $84.0 \%$ with serum cholesterol $<165 \mathrm{mg} / \mathrm{dL}$. These values are lower than the recommended standards for hemodialysis patients, and indicate the prevalence of malnutrition among hemodialysis patients at Dr. B. R. A. Memorial hospital raipur. Table also shows that $(20 \%)$ of hemodialysis patients had serum potassium level $>5.5 \mathrm{mEq} / \mathrm{L}$. Hyperkalemia is common in patients with CKD and when severe, can rapidly lead to death from cardiac arrest or paralysis of muscles that control ventilation. Therefore, control of serum potassium is a critically important part of dietary management in patients with $\mathrm{CKD}(\mathrm{NKF} / \mathrm{KDOQI}$, 2008).

\section{Table 2: Distribution of the study population by biochemical indicators of malnutrition}

\begin{tabular}{|c|c|c|c|c|}
\hline S. No. & Biochemical Tests & Abnormal Values & Frequency (50) & Percentage $(100 \%)$ \\
\hline 1. & Albumin & $<4.0 \mathrm{~g} / \mathrm{dL} / \mathrm{e}$ & $29 n t$ & $58 \%$ \\
\hline 2. & Creatinine & $<10 \mathrm{mg} / \mathrm{dL}$ & 32 & $64 \%$ \\
\hline 3. & Cholesterol & $<165 \mathrm{mg} / \mathrm{dL}$ & 42 & $84 \%$ \\
\hline 4. & Potassium & $>5.5 \mathrm{mEq} / \mathrm{L}<430$ & 10 & $20 \%$ \\
\hline
\end{tabular}
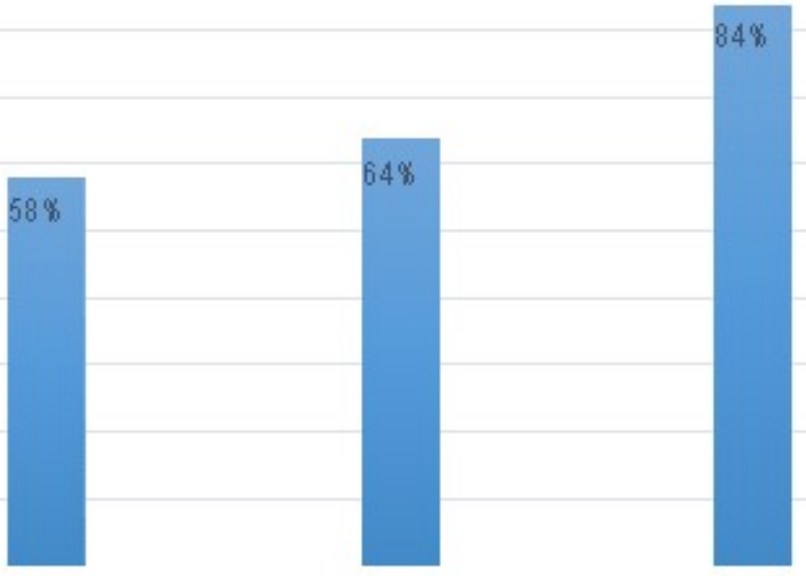

Figure: Showing Biochemical indicators of malnutrition (Albumin, Creatinine, and Cholesterol)

\begin{tabular}{|l|l|l|l|}
\hline Biochemical tests & Albumin $<4.0 \mathrm{~g} / \mathrm{dL}$ & Creatinine $<10 \mathrm{mg} / \mathrm{dL}$ & $<165 \mathrm{mg} / \mathrm{dL}$ Cholesterol \\
\hline
\end{tabular} 


\section{Conclusions And Recommendations}

Malnutrition is common among hemodialysis patients at Dr. B. R. A. Memorial Hospital Raipur and closely related to morbidity and mortality.

Approximately two thirds of the patients showed a biochemical malnutrition indicators, these include:

A. Hypoalbuminemia (58.0\%),

B. Low predialysis serum creatinine level $(64.0 \%)$,

C. Low serum cholesterol level $(84.0 \%)$,

D. And $60 \%$ of the patients had BMI less than the recommended BMI $\left(23.8 \mathrm{~kg} / \mathrm{m}^{2}\right)$ for hemodialysis patients.
$>$ There was a significant negative correlation between number of visits to ER and the number of admission days to hospitals over a year with serum albumin level, and BMI. The data suggests that the patients are at a high risk of morbidity and mortality.

There were a significant positive correlations between dietary protein, phosphorous, potassium intake with serum albumin level, serum phosphorous level, serum potassium level respectively. Our results showed that, hemodialysis patients need to decrease consumption of (phosphorous, potassium rich foods) and to increase dietary protein intake, to improve their nutritional status and to reduce CKD complications.

Gastrointestinal symptoms lead to inadequate food intake and may interfere significantly with the patients nutritional status.

at DR. B. R. A. Memorial Hospital:

A. Anemia - male $-62.0 \%$ - female $-38.0 \%$

B. Hypertension $(72.0 \%)$,

C. High turnover bone disease -hypocalcaemia$72.0 \%$ hypophosphatemia $-72.0 \%$

D. Hyperkalemia $(20.0 \%)$,

E. Diabetes mellitus (36\%).

And the presence of this co-morbidity has a significant adverse impact on patients survival.

There was a significant positive correlation between patients age (yrs), marital status, and monthly income (NIS) with BMI. The data suggests that demographic socioeconomic factors could contribute to a higher percentage of malnutrition.
The majority $(56.0 \%)$ of hemodialysis patients didn't have any diet regimen and about $(44.0 \%)$ of patients deviated from their fluid restrictions.

\section{Abbreviations:-}

UPS 2 Proteasome-ubiquitin system

CKD $\quad$ Chronic kidney disease

ESRD End-stage renal disease

HBV

IS

pCS

$\mathrm{HCl}$

$\mathrm{H} 2 \mathrm{SO} 4$

$\mathrm{H} 3 \mathrm{PO} 4$

$\mathrm{NaHCO} 3$

TWEAK

IL-1

IL-6
High biological value Indoxyl sulfate

p-Cresyl sulfate

Hydrogen chloride

Sulfuric acid

Phosphoric acids

Sodium bicarbonate

NF-related weak inducer of apoptosis

Interleukin-1

Interleukin-6 